\section{Tempo até o transplante e sobrevida em pacientes com insuficiência renal crônica no Estado do Rio de Janeiro, Brasil, 1998-2002}

\author{
Time to kidney transplantation in chronic renal \\ failure patients in the State of Rio de Janeiro, \\ Brazil, 1998-2002
}

\author{
${ }^{1}$ Escola Nacional de Saúde \\ Pública Sergio Arouca, \\ Fundação Oswaldo Cruz, Rio \\ de Janeiro, Brasil. \\ 2 Instituto de Medicina \\ Social, Universidade do \\ Estado do Rio de Janeiro, Rio \\ de Janeiro, Brasil. \\ 3 Centro de Informação \\ Científica e Tecnológica \\ Fundação Oswaldo Cruz, Rio \\ de Janeiro, Brasil. \\ ${ }^{4}$ Coordenação de \\ Fiscalização Sanitária \\ Secretaria de Estado de Saúde \\ do Estado do Rio de Janeiro, \\ Rio de Janeiro, Brasil. \\ Correspondência \\ C. B. Cunha \\ Departamento de \\ Epidemiologia e Métodos \\ Quantitativos em Saúde, \\ Escola Nacional de Saúde \\ Pública Sergio Arouca, \\ Fundação Oswaldo Cruz. \\ Av. Leopoldo Bulhões 1480, \\ Rio de Janeiro, $R J$ \\ 21041-210, Brasil. \\ cynthia.cunha@ensp.fiocruz.br
}

\begin{abstract}
This study analyzes the characteristics of 14,419 chronic renal failure patients treated with hemodialysis and time to first kidney transplantation in the State of Rio de Janeiro, Brazil, from 1998 to 2002. Survival analysis methods were used, such as the Kaplan-Meier non-parametric method and the semi-parametric method with the Cox proportional hazards model. Besides the survival model for transplantation, time to death was analyzed to compare the two models' estimates. During the period studied, only $6.3 \%$ of patients received transplants, $32.4 \%$ were referred for transplantation, and 6.3\% were included on the waiting list. Odds of transplantation were greater for those who had been referred, those on the waiting list, and younger patients. Diabetes mellitus reduced the probability of conducting transplantation by 35\%. All the estimates showed directions opposite to those obtained for the mortality survival model.

Survivorship (Public Health); Kidney Transplantation; Chronic Renal Insufficiency; Health Surveillance
\end{abstract}

\author{
Cynthia Braga da Cunha 1 \\ Antônio Carlos Ponce de León 2 \\ Joyce Mendes de Andrade Schramm 1 \\ Marilia Sá Carvalho 1 \\ Paulo Roberto Borges de Souza Júnior 3 \\ Reinaldo Chain 4
}

\section{Introdução}

Uma das doenças crônicas que requerem tratamento de alto custo do Sistema Único de Saúde (SUS) é a insuficiência renal crônica, que é causada principalmente pela hipertensão arterial e a diabetes mellitus 1,2. Existem duas alternativas de tratamento para os pacientes com insuficiência renal crônica, a diálise ou o transplante. A diálise é o procedimento mais utilizado, porém uma das modalidades de tratamento e reabilitação mais recomendadas para pacientes com insuficiência renal crônica é o transplante, pois oferece melhor qualidade de vida ao paciente, uma possível redução do risco de mortalidade, dependendo das características do paciente e menor custo que a diálise $3,4,5,6,7,8,9,10,11,12$. No entanto, Mazzuchi et al. 10 ponderam que a escolha do tratamento deveria levar em conta as características individuais dos pacientes, ou seja, fatores demográficos e de co-morbidade, pois cada modalidade de tratamento possui vantagens e desvantagens.

Mazzuchi et al. 13, no Uruguai, analisaram a sobrevida de pacientes em hemodiálise e de pacientes transplantados. Ao fim, no grupo de pacientes não diabéticos, não se encontrou diferença no tempo de sobrevida entre as duas modalidades de tratamento quando ajustado por fatores de co-morbidade. Entretanto, no grupo de diabéticos as taxas de sobrevida foram maiores para quem fez transplante renal. 
Para a realização do transplante são definidos critérios que incluem avaliações médica, cirúrgica e psicossocial do paciente com a realização de exames e a verificação da compatibilidade com o possível doador em relação aos Antígenos Leucocitários Humanos (HLA). As contra-indicações absolutas e relativas que não permitem a indicação do paciente como candidato potencial para o transplante renal são: infecção ativa, prognóstico de sobrevida é baixo, problemas psiquiátricos graves, psicose pobremente controlada, dependentes químicos, doença coronariana, hepatite ativa, úlcera péptica ativa, doença cérebro-vascular e possível não adesão do paciente ao tratamento. Não estão incluídas entre as contra-indicações: a idade avançada, o paciente ter sido transplantado anteriormente e nem o tipo de doença renal de base 14

No ano de 2003, segundo a Sociedade Brasileira de Nefrologia 15 , o número de pessoas com insuficiência renal, no Brasil, era na ordem de 54 mil e destas perto de 48 mil recorrem à hemodiálise, e o número de transplantes realizados no ano de 2003 foi de apenas 3.025 em todo o país. Portanto, apenas $6,2 \%$ dos pacientes com insuficiência renal crônica realizaram o procedimento de transplante em todo o Brasil.

No Brasil, de 1995 a 2002, o número de transplantes realizados teve um aumento de $94,3 \%$ segundo o Ministério da Saúde 16. Tal aumento pode ser explicado por um possível crescimento da captação de órgãos em razão de uma maior conscientização da sociedade brasileira e o crescimento do acesso ao tratamento no período.

Um estudo qualitativo mostrou que existe o problema da recusa das famílias de autorizarem a retirada de órgãos de pessoas com morte cerebral relatando que cerca de metade das famílias negam a doação. $\mathrm{O}$ autor relata que os principais motivos são a falta de explicação à família, por parte do médico, do que é morte cerebral, medo da comercialização de órgãos e assistência médica, englobando o atendimento médico e hospitalar, julgada inadequada 17 .

O cenário mundial também apresenta pouca oferta de órgãos e um grande número de indivíduos indicados para o transplante renal, trazendo com isso um grande cuidado em relação à escolha dos critérios de indicação para transplante. Geralmente, o médico realiza um levantamento da história médica e psicossocial do paciente além de exames para avaliar se um paciente deve ser indicado ou não 18.

A Portaria GM no. 3.407/1998 19 do Ministério da Saúde trata dos critérios de elegibilidade e de exclusão para os pacientes inscritos na lista de espera para o transplante renal. Como critérios excludentes listam-se a amostra do soro do re- ceptor fora do prazo de validade e a incompatibilidade sangüínea entre o doador e receptor, em relação ao sistema ABO. Os critérios classificatórios são a compatibilidade em relação ao HLA, idade do receptor, tempo decorrido da inscrição na lista única e a indicação de transplante combinado de rim e pâncreas. O indivíduo somente pode se inscrever na lista de espera se for indicado pelo médico. No Brasil, o tratamento é oferecido pelo SUS e planos de saúde privados por meio da regulamentação da Agência Nacional de Saúde Suplementar (ANS).

Este estudo tem como principal objetivo a análise do tempo transcorrido até a primeira realização do transplante e sobrevida dos pacientes com insuficiência renal crônica tratados por hemodiálise no Estado do Rio de Janeiro no período de 1998 a 2002.

\section{Metodologia}

A fonte de dados é o Sistema de Autorizações de Procedimentos de Alta Complexidade (APAC), criado pela Portaria GM no. 2.043/1996 20 do Ministério da Saúde para os serviços de Terapia Renal Substitutiva (TRS), com financiamento dado pelo SUS. Este registro nacional gera um banco de dados do Sistema de Informações Ambulatoriais do Sistema Único de Saúde (SIA-SUS) que contém as autorizações de procedimentos de alta complexidade referentes ao tratamento por diálise de pacientes com insuficiência renal crônica.

A APAC engloba diversos arquivos, cada um com a descrição de aspectos específicos, tais quais dados de cada paciente, dados relativos aos procedimentos, exames realizados em cada paciente e características das unidades de diálise. Todos esses arquivos são gerados mensalmente e o paciente é identificado por um número; com este número e a data de emissão do registro procede-se ao relacionamento de todos os bancos.

O tempo até a realização do primeiro transplante e o tempo até o óbito do paciente foram analisados pela utilização de técnicas de análise de sobrevida, em que o período de tempo de cada paciente será visto como um processo de contagem $21,21,23$, um processo estocástico em que as informações para um indivíduo são apresentadas com múltiplas observações, com cada observação referente a um tempo $t$. A data de entrada do paciente foi o início do intervalo de tempo em risco. No nosso estudo, a data do primeiro registro de tratamento por hemodiálise realizado no centro de diálise e a data marcadora do fim do seguimento será o final do intervalo de tempo em risco, isto é, a data do último registro (censura 
ou primeiro transplante) realizado no período de janeiro de 1998 a dezembro de 2002. Para a modelagem do tempo até o óbito construiu-se outro banco no qual se acompanhou o indivíduo até o óbito ou até a data do último registro.

Nos dados utilizados neste estudo ocorrem dois tipos de problemas que podem fornecer estimativas enviesadas: truncamento à esquerda (pacientes de sobrevida longa) e censura à esquerda. O primeiro ocorre quando a entrada do paciente no sistema ocorreu antes de janeiro de 1998. O segundo tipo quando indivíduos vão a óbito ou são transplantados pela primeira vez antes do início do período de observação. Em ambos os problemas, a estimativa de risco é superestimada para tempo até o óbito e subestimada para o tempo até o transplante 24,25 .

O processo de contagem utilizado neste estudo permite o tratamento de dados referentes aos pacientes em terapia renal substitutiva, registrados na APAC, pois o grupo de risco é formado apenas por pacientes que entraram quando a observação teve início em janeiro de 1998 e não quando o paciente começou a diálise. A inferência torna-se válida apenas nesta janela de cinco anos de observação. Além disso, como é uma coorte aberta, o viés por tempos de sobrevidas maiores é controlado pela entrada e saída de indivíduos a todo instante. Contudo, para truncamento à esquerda, este estudo não utilizou nenhuma técnica especial de modelagem, adotando como premissa que nenhuma mudança das condições geradoras desse processo tenha ocorrido.

Inicialmente, empregou-se a análise univariada com o uso da técnica não paramétrica de Kaplan-Meier para visualizar o padrão de sobrevida total e verificar a existência de diferença entre os grupos, depois se utilizou a modelagem semiparamétrica com o uso do modelo de riscos proporcionais de Cox 26,27. A premissa de risco relativo constante no tempo foi avaliada por meio da análise de resíduos de Schoenfeld. As variáveis selecionadas para análise foram: sexo, idade, patologia que originou a doença renal - diabetes mellitus/doença congênita/doença renal/outras doenças - sendo doença congênita a categoria de base, indicação de transplante e inscrição na lista de espera. Além do modelo de sobrevida para o primeiro transplante, o tempo até o óbito também foi estimado para a comparação das estimativas dos dois modelos. No modelo do tempo até o primeiro transplante na variável idade do paciente aplicou-se uma spline cúbica (aproximação polinomial cúbica), de forma a permitir maior flexibilidade da forma da variável e preencher a premissa de linearidade da parte paramétrica do modelo de Cox. O nível de significância adotado é de 5\%. O programa de computador utilizado foi R 2.1.0 (R Development Core Team, Viena, Áustria).

\section{Resultados}

A Tabela 1 apresenta características do paciente segundo as diferentes patologias que levaram à ocorrência da doença renal. Do total de 14.419 pacientes, 55,3\% são do sexo masculino, 32,4\% foram indicados para o transplante e apenas $6,3 \%$ inscreveram-se na lista de espera para o transplante de rim. O principal motivo de cobrança foi a permanência com o mesmo procedimento de hemodiálise representando $60,1 \%$, e o segundo maior motivo é o óbito pela doença renal e por outras causas, ambos somados representando mais de $90 \%$. Quanto à faixa etária, 78,8\% dos pacientes estão abaixo de 65 anos e, no Estado do Rio de Janeiro, as principais patologias causadoras da insuficiência renal crônica são a hipertensão, a doença renal e a diabetes mellitus, e estas três englobam 79,9\% dos pacientes.

Observou-se que quanto maior a idade, maior é a ocorrência de hipertensão e diabetes mellitus, excetuando a doença congênita com maior ocorrência, $60,2 \%$, na faixa etária de 45 a 64 anos. A doença renal, quando comparada com diabetes mellitus e hipertensão, apresenta freqüências 13,2 e 4,2 vezes maiores, respectivamente, na faixa etária de menores de vinte anos.

Adicionalmente, encontrou-se uma maior proporção relacionada à indicação para o transplante ocorrendo entre os pacientes não diabéticos e, especialmente, para os portadores de hipertensão arterial com $37,4 \%$ de pacientes indicados. Para os inscritos na lista de espera, os diabéticos são em menor número quando comparados aos não inscritos. A freqüência do procedimento do primeiro transplante é menor entre os diabéticos, com 1,7\%, é maior para os que tiveram doença renal apresentando 9,8\%, enquanto a mortalidade é maior para os primeiros, com $42,7 \%$, apresentando uma ocorrência 1,6 vezes maior em relação aos últimos.

A Tabela 2 apresenta o modelo de riscos proporcionais de Cox aplicado aos desfechos de tempo até a primeira realização do transplante e tempo até o óbito com variáveis preditoras, sendo a idade, as patologias que originaram a doença renal, indicação e inscrição na lista de espera para transplante. Para o modelo do primeiro transplante, mostraram-se significativas a idade, todas as patologias, indicação e inscrição na lista de espera para o transplante. Para a idade há redução no risco, mostrando que quanto maior a idade, maior é a probabilidade de não realizar 
Tabela 1

Características dos pacientes segundo a patologia que originou a doença renal nos pacientes portadores de insuficiência renal crônica tratados por hemodiálise no Estado do Rio de Janeiro, Brasil, 1998-2002.

\begin{tabular}{|c|c|c|c|c|c|c|}
\hline \multirow[t]{2}{*}{ Variáveis * } & \multicolumn{5}{|c|}{ Patologia que originou a doença renal (\%) ** } & \multirow[t]{2}{*}{ Total } \\
\hline & Diabetes & Hipertensão & Doença renal & Doença congênita & Outros & \\
\hline Sexo masculino & 52,1 & 56,8 & 56,3 & 50,1 & 53,8 & 55,3 \\
\hline \multicolumn{7}{|l|}{ Faixa etária (anos) } \\
\hline$<20$ & 0,5 & 1,6 & 6,7 & 0,8 & 4,3 & 3,0 \\
\hline $20-44$ & 14,7 & 33,4 & 40,1 & 22,4 & 36,7 & 32,4 \\
\hline $45-64$ & 55,9 & 43,8 & 35,2 & 60,2 & 39,8 & 43,3 \\
\hline $65-74$ & 23,5 & 14,0 & 12,4 & 10,1 & 13,5 & 14,9 \\
\hline 75 e mais & 5,3 & 7,2 & 5,7 & 6,4 & 5,7 & 6,3 \\
\hline \multicolumn{7}{|l|}{ Procedimento } \\
\hline Alta para transplante & 1,7 & 5,9 & 9,8 & 8,4 & 6,3 & 6,3 \\
\hline Alta por abandono de tratamento & 0,5 & 0,9 & 0,6 & 0,6 & 0,6 & 0,7 \\
\hline Permanência na diálise & 50,9 & 61,2 & 60,0 & 65,8 & 64,3 & 60,1 \\
\hline Transferência por intercorrência & 4,2 & 2,7 & 2,6 & 3,4 & 2,4 & 2,9 \\
\hline Óbito & 42,7 & 29,3 & 27,0 & 21,8 & 26,3 & 30,1 \\
\hline Indicação para transplante & 26,1 & 37,4 & 31,5 & 32,8 & 27,1 & 32,4 \\
\hline Inscrição na lista de espera & 1,8 & 7,0 & 7,7 & 6,4 & 6,6 & 6,3 \\
\hline Total (N) & 2.170 & 6.053 & 3.300 & 357 & 2.539 & 14.419 \\
\hline
\end{tabular}

Fonte: Sistema de Autorizações de Procedimentos de Alta Complexidade (DATASUS/Ministério da Saúde).

* Dados oriundos do bando de dados de pacientes observados até a ocorrência do primeiro transplante;

** $p$ valor $<0,001$ para todas as características.

Tabela 2

Modelos de riscos proporcionais de Cox para óbito e tempo até o primeiro transplante. Estado do Rio de Janeiro, Brasil 1998-2002.

\begin{tabular}{|c|c|c|}
\hline \multirow[t]{2}{*}{ Variáveis } & \multicolumn{2}{|c|}{ RR (IC95\%) } \\
\hline & Óbito * & Transplante ** \\
\hline \multicolumn{3}{|l|}{ Idade } \\
\hline 1ㅇ termo & $1,04(1,04-1,04)$ & $0,98(0,97-0,99)$ \\
\hline 2o termo *** & - & $0,93(0,89-0,97)$ \\
\hline 3o termo *** & - & $0,92(0,66-1,30)$ \\
\hline \multicolumn{3}{|c|}{ Patologia que originou a doença renal } \\
\hline Diabetes & $1,56(1,43-1,70)$ & $0,65(0,46-0,92)$ \\
\hline Renal & $0,97(0,89-1,06)$ & $1,19(1,02-1,39)$ \\
\hline Congênita & $0,63(0,49-0,80)$ & $1,90(1,31-2,74)$ \\
\hline Outras causas & $1,10(0,99-1,21)$ & $0,94(0,78-1,14)$ \\
\hline Indicação para transplante & $0,85(0,78-0,92)$ & $1,34(1,15-1,56)$ \\
\hline Inscrição na lista de espera & $0,63(0,52-0,77)$ & $1,38(1,14-1,66)$ \\
\hline
\end{tabular}

* Dados oriundos do bando de dados de pacientes observados até a ocorrência de óbito;

** Dados oriundos do bando de dados de pacientes observados até a ocorrência do primeiro transplante;

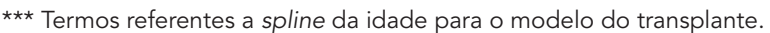


o procedimento de transplante. No modelo relativo ao óbito existe um aumento no risco de $4 \%$ a cada incremento de um ano, mostrando que quanto maior a idade, maior é o risco de óbito.

Nos pacientes em TRS para doença de base diabetes mellitus observou-se uma queda de $35 \%$ na probabilidade de realização de transplante. Todas as estimativas dos modelos utilizados para transplante e óbito apresentaram direções contrárias.

A Figura 1 apresenta os diferentes modos de entrada da idade no modelo. Ao comparar a forma da associação da idade, no gráfico superior a idade, entra linearmente para óbito e, no inferior, curvilinearmente para primeiro transplante, permitindo a flexibilidade desta variável quando ocorre um relacionamento não linear, observouse que a realização do transplante é estável até a idade de quarenta anos e, após esta idade, a queda do hazard é bastante acentuada.

As Figuras 2 e 3 apresentam a probabilidade de sobrevida e de não ser transplantado, respectivamente, segundo quantis (com variação de 0,20 ) da idade ajustado por outras co-variáveis do modelo. Claramente, verifica-se o efeito da idade tanto para o óbito quanto para o primeiro transplante em qualquer que seja o status do indivíduo quanto à patologia.

\section{Discussão}

Os achados deste estudo apontam a grave situação dos pacientes com insuficiência renal crônica no Estado do Rio de Janeiro. Os portadores de insuficiência renal crônica são afetados tanto pelos problemas que o setor de diálise enfrenta, no Brasil, devido ao baixo valor repassado aos prestadores de serviço pelo SUS, responsável por $95 \%$ do atendimento ao paciente em diálise 28 , como pelo pequeno número de transplantes realizados em vista da população com insuficiência renal crônica existente. A primeira opção de TRS deveria ser o transplante renal com o paciente sem ter realizado nenhuma sessão de hemodiálise e outra questão é a estrutura de serviços para captação e doação de órgãos, uma vez que é de conhecimento geral que os doadores, em sua grande maioria, são familiares dos pacientes.

As taxas de transplante possuem uma grande variação mundial, indo de 0,3 por milhão em Bangladesh a 61,0 por milhão na Catalunha, Espanha, e, por faixa etária, as crianças são as detentoras de maior número de transplantes na Holanda, Finlândia e Noruega. Para os idosos, ao compararem-se as taxas dos países, a Noruega realiza cinco vezes mais transplantes do que os outros 18 .
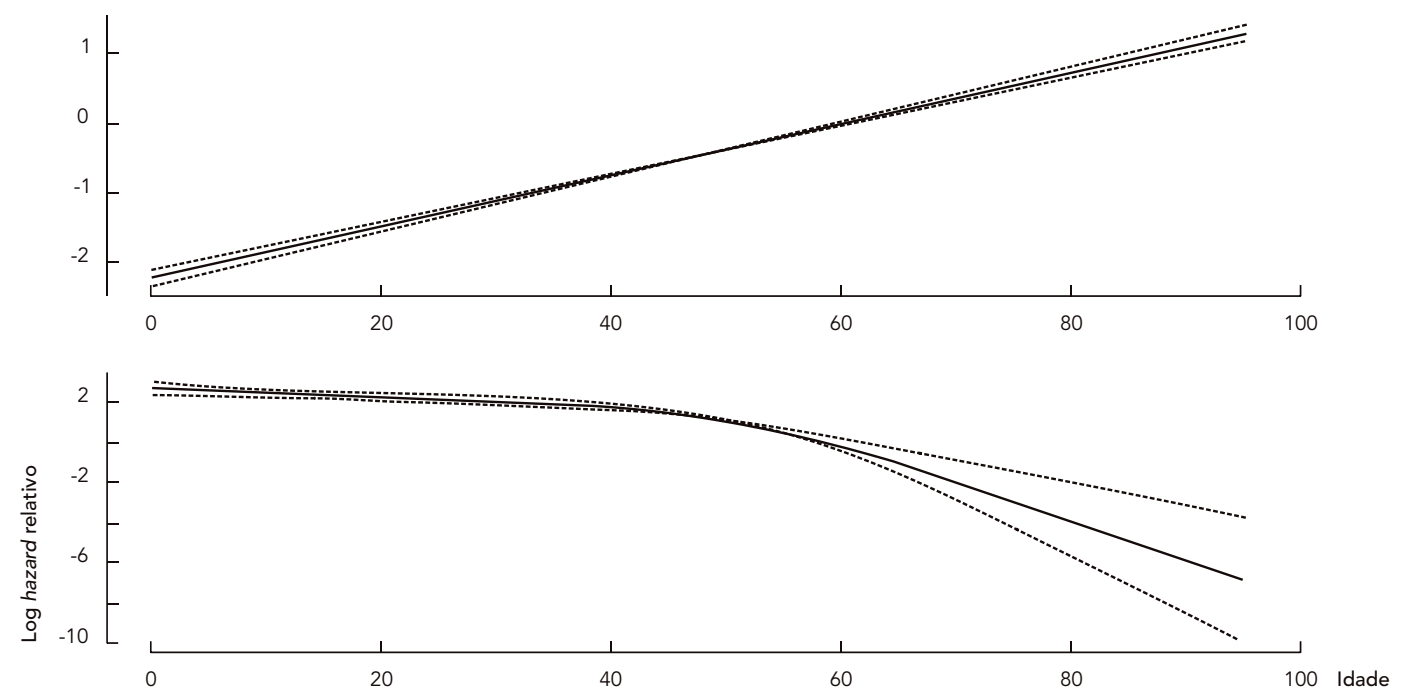

Ajustado por paciente com doença congênita, não indicado e não inscrito. 
Probabilidade de sobrevida estimada pelo modelo de riscos proporcionais de Cox para tempo até o óbito para quantis da idade ajustada por co-variáveis. Estado do Rio de Janeiro, Brasil, 1998-2002.
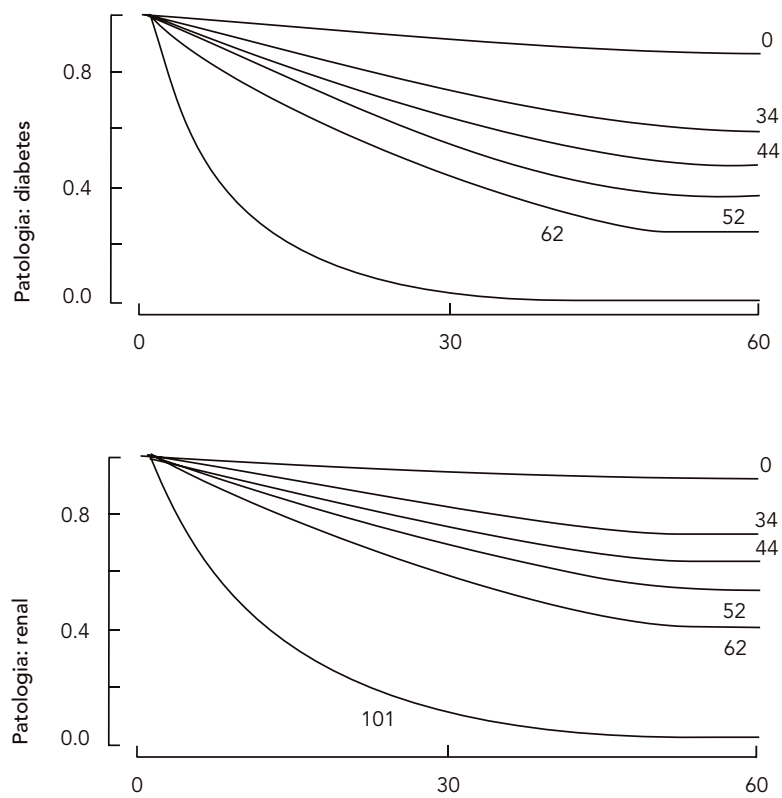
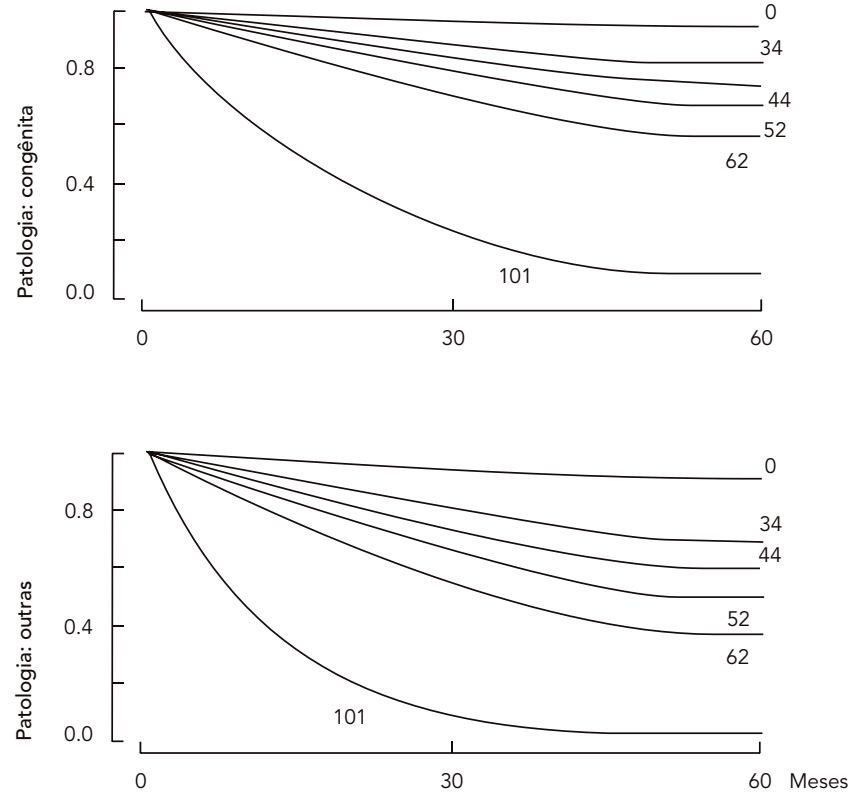

Ajustado por paciente não indicado e não inscrito.

Nos Estados Unidos, no ano de 1997, 28,4\% dos pacientes viviam com um rim funcional mesmo em um cenário de pouca oferta de órgãos. Este país apresenta uma tendência crescente da incidência de insuficiência renal crônica ao longo dos anos, sobretudo, entre os afro-americanos, idosos e diabéticos. É justamente nestes dois últimos grupos que a mortalidade é maior e a indicação médica é mais difícil de ser dada pelas possíveis complicações que podem acompanhá-los 29.

No Estado do Rio de Janeiro, a maioria dos pacientes foi tratada por hemodiálise e apenas uma minoria de $6,3 \%$ conseguiu o transplante renal no período de 1998 a 2002. Em análises suplementares, encontrou-se que os indivíduos indicados e inscritos na lista de espera para o transplante possuem uma maior chance de serem transplantados, embora apenas $19,3 \%$ dos indivíduos inscritos conseguiram o transplante e, entre os indicados, apenas $10,1 \%$ foram transplantados. Observou-se a existência de um grande contingente de pessoas transplantadas que não foram inscritas e nem indicadas $(59,3 \%)$ pelo especialista para o transplante. Tais achados apontam, por um lado, a grande fragilidade do sistema de transplante e, por outro, a necessidade de garantir uma melhor qualidade no sistema de registro dos pacientes pelo sistema APAC. Os idosos, aqueles com idade maior ou igual que 65 anos, representaram $21,2 \%$ dos pacientes, apresentando uma menor indicação para transplante e uma maior ocorrência de diabetes mellitus como a patologia que originou a doença renal.

A diabetes mellitus aparece nas estimativas como forte limitadora do transplante seguida da hipertensão arterial, patologias altamente prevalentes nos idosos, o que em tese corrobora a hipótese de que os idosos teriam menos chance de serem transplantados. Na população estudada, $15 \%$ dos pacientes tratados eram portadores de diabetes mellitus. Este agravo ocupou os primeiros lugares entre as principais causas de Disability Adjusted Life Years (DALY) 30 tanto entre os homens, quanto nas mulheres. Segundo United States Renal Data System (USRDS) 18, as taxas de insuficiência renal crônica aumentam em todo o mundo e o diabetes mellitus é uma importante causa da IRC, tornando necessárias ações preventivas e de detecção quanto a esta doença. 
Probabilidade de sobrevida estimada pelo modelo de riscos proporcionais de Cox para tempo até o primeiro transplante para quantis da idade ajustada por co-variáveis. Estado do Rio de Janeiro, Brasil, 1998-2002.
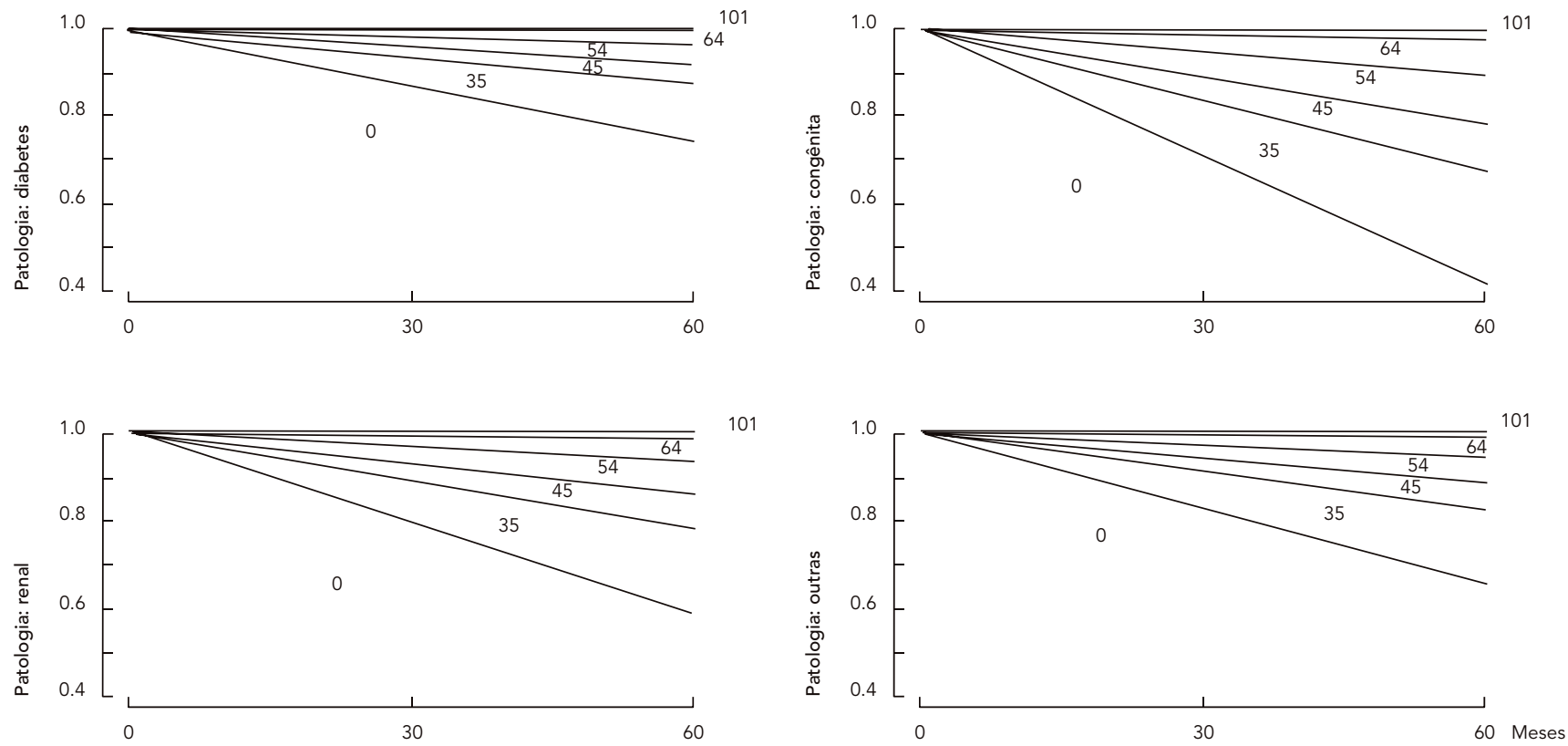

Ajustado por paciente não indicado e não inscrito.

A idade também consta como um dos critérios de ranqueamento na lista de espera do transplante renal para a escolha do indivíduo, ainda que, segundo Ramos et al. 14, a idade não deveria ser um dos critérios de seleção para o transplante. Os aspectos éticos devem ser avaliados cuidadosamente tendo em vista a relevância de considerar a esperança de vida do paciente que, se fosse menor que a duração da vida útil do órgão, o paciente em questão não seria escolhido 31 .

Vale ressaltar que não foi possível incorporar as informações referentes às co-morbidades na análise realizada por conta da inexistência de campos para o seu registro na base de dados utilizada. A literatura relata que, geralmente, os pacientes escolhidos para o transplante possuem um estado de saúde melhor e uma faixa etária mais jovem dos que estão no processo de hemodiálise 9,32.

A situação no Estado do Rio de Janeiro pode ser considerada bastante grave segundo os resultados aqui apresentados, fato que aponta para a importância de implantar ações de regulação do setor de hemodiálise e transplante, tendo em vista o crescimento anual das necessidades e do alto custo dos procedimentos. 


\section{Resumo}

Neste estudo, descreveram-se as características dos 14.419 pacientes com insuficiência renal crônica tratados por hemodiálise no Estado do Rio de Janeiro, Brasil, e analisou-se o tempo até a primeira realização do transplante no período de 1998 a 2002. Técnicas de análise de sobrevida como a análise não paramétrica de Kaplan-Meier e a modelagem semiparamétrica com o modelo de riscos proporcionais de Cox foram utilizadas. Além do modelo de sobrevida para transplante, o tempo até o óbito foi estimado para a comparação das estimativas dos dois modelos. Os resultados mostraram que, no período estudado, apenas 6,3\% dos pacientes foram transplantados, 32,4\% foram indicados e 6,3\% inscritos na lista de espera. Observa-se que a probabilidade de transplante dos pacientes indicados, inscritos para o transplante e os que estão em uma faixa etária reduzida é maior. A diabetes mellitus possui um efeito redutor de $35 \%$ no risco de realização de transplante. Todas as estimativas apresentaram direções contrárias às obtidas pelo modelo de sobrevida para óbito.

Sobrevida; Transplante de Rim; Insuficiência Renal Crônica; Vigilância Sanitária

\section{Referências}

1. Bruno RM, Gross JL. Prognostic factors in Brazilian diabetic patients starting dialysis: a 3.6-year follow-up study. J Diabetes Complications 2000; 14:266-71.

2. Klag MJ, Whelton PK, Randall BL, Neaton JD, Brancati FL, Ford, CE, et al. Blood pressure and endstage renal disease in men. N Engl J Med 1996; 334:13-8.

3. Bradley JR, Evans DB, Calne RY. Long-term survival in haemodialysis patients. Lancet 1987; 7:295-6.

4. Bremer BA, McCauley CR, Wrona RN, Johnson JP. Quality of life in end-stage renal disease: a reexamination. Am J Kidney Dis 1989; 13:200-9.

5. Churchill DN, Torrance GW, Taylor DW, Barnes CC, Ludwin D, Shimizu A, et al. Measurement of quality of life in end-stage renal disease: the time tradeoff approach. Clin Invest Med 1987; 10:14-20.

6. Harris SAC, Brown EA. Patients surviving more than 10 years on haemodialysis. The natural history of the complications of the treatment. Nephrol Dial Transplant 1998; 13:1326-33.

7. Karlberg I, Nyberg G. Cost-effectiveness studies of renal transplantation. Int J Technol Assess Health Care 1995; 11:611-22.

8. Mailloux LU, Henrich WL. Patient survival and the adequacy of maintenance dialysis. http://patients. uptodate.com (acessado em 25/Set/2003).

9. Mandigers CM, de Jong W, van den Wall Bake AW Gerlag PG. Renal replacement therapy in the elderly. Neth J Med 1996; 49:135-42.

10. Mazzuchi NM, Fernández-Cean JM, Carbonell E. Criteria for selection of ESRD treatment modalities. Kidney Int 2000; 50:136-43.

\section{Colaboradores}

C. B. Cunha realizou pesquisa bibliográfica, análise dos dados, discussão dos resultados e redação do manuscrito. A. C. P. Leon colaborou na análise dos dados e discussão dos resultados. J. M. A. Schramm participou da concepção do estudo, discussão dos resultados e redação do manuscrito. M. S. Carvalho participou da concepção do estudo, análise dos dados e discussão dos resultados. P. R. B. Souza Júnior participou da análise dos dados e discussão dos resultados. R. Chain participou da concepção do estudo e discussão dos resultados.

\section{Agradecimentos}

Ao Centro de Vigilância Sanitária do Estado do Rio de Janeiro, Secretaria do Estado de Saúde do Estado do Rio de Janeiro e a Fundação Carlos Chagas Filho de Amparo à Pesquisa do Estado do Rio de Janeiro.
11. Port FK, Wolfe RA, Mauger EA, Berling DP, Jiang K. Comparison of survival probabilities for dialysis patients vs. cadaveric renal transplant recipients. JAMA 1993; 270:1339-43.

12. Schnuelle P, Lorenz D, Trede M, van der Woude FJ. Impact of renal cadaveric transplantation on survival in end-stage renal failure: evidence for reduced mortality risk compared with hemodialysis during long-term follow-up. J Am Soc Nephrol 1998; 9:2135-41.

13. Mazzuchi NM, González-Martínez F, Carbonell E Fernández-Cean JM, Curi L, Orihuela S. Comparison of survival for haemodialysis patients vs. renal transplant recipients treated in Uruguay. Nephrol Dial Transplant 1999; 14:2849-54.

14. Ramos E, Sayegh MH, Brennam DC. Evaluation of the potential renal transplant recipient. http://patients.uptodate.com/ (acessado em 16/Abr/2004).

15. Sociedade Brasileira de Nefrologia. Censo geral. http://www.sbn.org.br/censos.htm (acessado em $17 / \mathrm{Jul} / 2005)$

16. Ministério da Saúde. Central nacional. Sistema nacional de transplantes. http://dtr2001.saude.gov. br/transplantes/Site_SNT_arquivos/CNNCDO. htm (acessado em 17/Jul/2005).

17. Meira GS. Fio de esperança: avaliação da doação de órgãos cadavéricos para transplantes no norte de Minas Gerais [Tese de Doutorado]. São Paulo: Universidade Federal do Estado de São Paulo; 2002.

18. United States Renal Data System. USRDS 2002 an nual data report. Bethesda: National Institutes of Health; 2002. 
19. Brasil. Portaria GM no. 3.407. Diário Oficial da União 1998; 6 ago.

20. Brasil. Portaria GM no. 2.043. Diário Oficial da União 1996; 14 out.

21. Fleming TR, Harrington DP. Counting processes and survival analysis. New York: Wiley; 1991.

22. Therneau TM, Grambsch P. Modeling survival data - extending the Cox model. New York: SpringerVerlag; 2000.

23. Andersen PK, Bogan O, Gill RD, Keiding N. Statistical models based on counting processes. New York: Springer-Verlag; 1993.

24. Wang MC, Brookmeyer R, Jewell NP. Statistical models for prevalent data. Biometrics 1993; 49: $1-11$.

25. Sousa IPSC. Risk factors and centre effects in survival of haemodialysis patients [Master Thesis]. Lancaster: University of Lancaster; 2002.

26. Cox DR, Oakes D. Analysis of survival data. London: Chapman \& Hall; 1984.
27. Harrell Jr. FE. Regression modeling strategies. New York: Springer-Verlag; 2002.

28. Associação Médica Brasileira. Hemodiálise em crise. http://www.amb.org.br/jamb/set_out01/ pg12.pdf (acessado em 15/Jul/2003).

29. Schwab SJ, Paul LC. Survival with end-stage renal failure in the 1990s. Curr Opin Nephrol Hypertens 1996; 5:477-9.

30. Escola Nacional de Saúde Pública, Fundação Oswaldo Cruz. Estimativa da carga de doença do Brasil - 1998. Relatório final. Rio de Janeiro: Escola Nacional de Saúde Pública, Fundação Oswaldo Cruz; 2002.

31. Reikes ST. Trends in end-stage renal disease. Postgrad Med 2000; 108:124-42.

32. United Network for Organ Sharing. UNOS ethics committee - general considerations in assessment for transplant candidacy. http://www.unos.org/ Resources/bioethics.asp?index $=4$ (acessado em 17/Jul/2005).

Recebido em 18/Jul/2005

Versão final reapresentada em 15/Mai/2006

Aprovado em 11/Ago/2006 